\title{
A Quadratic Interpolation-Based Variational Bayesian Algorithm for Measurement Information Lost in Underwater Navigation
}

\author{
Yuan Yang $\mathbb{D},{ }^{1}$ Jiacheng Tang, ${ }^{2}$ Haoqian Huang, ${ }^{2}$ Xiaoguo Zhang, ${ }^{1}$ Tingting Zhang, \\ and Yujin Kuang ${ }^{1}$ \\ ${ }^{1}$ Key Laboratory of Micro-Inertial Instrument and Advanced Navigation Technology, Ministry of Education, \\ School of Instrument Science and Engineering, Southeast University, Nanjing 210096, China \\ ${ }^{2}$ College of Energy and Electrical Engineering, Hohai University, Nanjing 210098, China \\ ${ }^{3}$ College of Command and Control Engineering, Army Engineering University, Nanjing 210007, China
}

Correspondence should be addressed to Yuan Yang; yangyuan@seu.edu.cn

Received 4 November 2020; Revised 1 December 2020; Accepted 14 December 2020; Published 23 December 2020

Academic Editor: Gaoge $\mathrm{Hu}$

Copyright (c) 2020 Yuan Yang et al. This is an open access article distributed under the Creative Commons Attribution License, which permits unrestricted use, distribution, and reproduction in any medium, provided the original work is properly cited.

The main challenges of sequential estimations of underwater navigation applications are the internal/external measurement noise and the missing measurement situations. A quadratic interpolation-based variational Bayesian filter (QIVBF) is proposed to solve the underwater navigation problem of measurement information missing or insufficiency. The quadratic interpolation is used to improve the observed vector for the precision and stability of sequential estimations when the environment is changed or the measurement information is lost. The state vector, the predicted error covariance matrix, and the measurement noise matrix are derived based on the variational Bayesian method. Simulation results demonstrate the superiority of the proposed QIVBF compared with the traditional algorithm under the condition of measurement information lost by autonomous underwater vehicles.

\section{Introduction}

Ocean has already become the strategic goal of many countries because of its underdeveloped resources, marine environments, and high-tech fields $[1,2]$. The autonomous underwater vehicles (AUVs) have become one of the important tools for underwater detection, environment survey, and underwater reconnaissance in the ocean $[3,4]$. The navigation and positioning methods with high accuracy for AUV are the necessary conditions to acquire effective information [5], and also, they are the key technologies to determine whether AUV can work normally and return back safely $[6,7]$. The more accurate navigation and positioning methods for AUV are the necessary conditions for the underwater vehicle to ensure the correct travel $[8,9]$.
The development of underwater vehicles faces many problems; for instance, the ocean environment is complex and changeable due to all sorts of noise interferences, and the AUV may fail to represent the true sensor measurements in many actual applications, like the presence of ocean current, salt cliffs, and ships around. The measurements are sometimes unobservable or insufficient so that positions calculated are erroneous [10-12]. On the other hand, a few navigation methods based on electromagnetic transmitting cannot be used underwater since the signal attenuates quickly underwater [13]. What is more, the localization error of the MEMS system accumulates along with time [14-17]. Those above weaknesses will cause the measurement missing, which has an adverse impact on the positioning of AUV [18]. However, the debugging and arranging process would waste time and material resources. Therefore, the 
performances of estimation algorithms are vital to working normally for AUV [19, 20].

The Kalman filter (KF), which is a widely and classical recursive filter, could provide the optimal state estimates in the linear dynamic system in the scenarios of known noise models and system models [21-24]. Additionally, the accurate measurements are difficult for the underwater integrated navigation system to describe because the performance of sensors varies with the change in the environment and measurement information probability lost $[25,26]$. The joint probability density function (PDF) is used to model the information of the AUV. Then, the information of the joint PDF is determined by using the variational Bayesian (VB) method [27] in recursive process, which is the optimal. To further improve the capability of the navigation with the missing measurements, some strategies are required.

The recently proposed variational Bayesian- (VB-) based adaptive KF is a deterministic approximate Bayesian method that transforms the solution of the posterior probability density function based on Bayes' theorem into the solution of the functional extreme value $[28,29]$. It has more ideal approximate estimation results and computational overhead $[30,31]$. It significantly reduces the difficulty of calculation and makes high-precision filtering possible [32-35].

Aiming to mitigate the underwater navigation problem of measurement information missing or insufficiency, this paper proposes a quadratic interpolation-based variational Bayesian filter (QIVBF) algorithm. The QIVBF makes better use of the quadratic interpolation (QI) method and the VB method, so that the predicted error covariance matrix and the measurement noise matrix are derived to estimate the state vector more accurately. The quadratic interpolation improves the observed vector for the precision and stability of sequential estimations when the environment is changed or the measurement information is lost.

The rest of the paper is presented as follows. In Section 2, the situation of the measurement lost in underwater navigation is described in detail. In Section 3, the QI method is used in measurement missing situations. In Section 4 , the VB method is used to estimate the accurately predicted error covariance matrix, measurement error matrix, and state vector. Section 5 shows the simulation in underwater navigation. Section 6 gives the main conclusions of the paper.

\section{Problem Statement}

An underwater navigation function with measurement information lost is described as follows [36]:

$$
\left\{\begin{array}{l}
\mathbf{X}_{k \mid k-1}=f_{k-1}\left(\mathbf{X}_{k-1 \mid k-1}\right)+\omega_{k-1}, \\
\mathbf{Z}_{k}=\mathrm{H}_{k} \mathbf{X}_{k \mid k}+v_{k}
\end{array}\right.
$$

where $\mathbf{X}_{k-1 \mid k-1}=\left[\begin{array}{llll}\widehat{P}_{x, k-1} & \widehat{V}_{x, k-1} & \widehat{P}_{y, k-1} & \widehat{V}_{y, k-1}\end{array}\right]^{T}$ is the state posterior given the observation at time $k-1 ; \widehat{P}_{x, k-1}$ and $\widehat{P}_{y, k-1}$ denote the positions of the AUV at the time $k-1$ in $x$ and $y$ directions, respectively. $\widehat{V}_{x, k-1}$ and $\widehat{V}_{y, k-1}$ denote the velocities of the AUV at the time $k-1$ in $x$ and $y$ directions, respectively. The $\mathbf{Z}_{k}=\left[\begin{array}{lllll}P_{x, k-1} & V_{x, k-1} & P_{y, k-1} & V_{y, k-1}\end{array}\right]^{T}$ is the measurement vector at the time $k$, with $P_{x, k-1}$ and $P_{y, k-1}$ representing the position measurements of the AUV at the time $k-1$ in $x$ and $y$ directions, respectively, and $V_{x, k-1}$ and $V_{y, k-1}$ are the velocity measurements of the AUV at the time $k-1$ in $x$ and $y$ directions, respectively. The $\omega_{k-1}$ denotes the process noise, which often follows the Gaussian distribution with zero mean vector and process error covariance $\mathbf{Q}_{k-1}$. Similarly, $v_{k}$ means the measurement noise, which follows the Gaussian distribution with zero mean vector and measurement error covariance $\mathbf{R}_{k}$. The $f_{k-1}(\cdot)$ is the known process function at the time $k-1$. The $\mathrm{H}_{k}$ is the measurement model at the time $k$.

However, the underwater environment is complex and time-varying. Hence, the measurement information is unavoidable lost, which is described as follows:

$$
\mathbf{Y}_{k}= \begin{cases}0, & \text { pro }=a, \\ \mathbf{Z}_{k}, & \text { pro }=1-a,\end{cases}
$$

where $\mathbf{Y}_{k}$ is the observation vector and pro means the probability.

\section{Cubature Unscented Kalman Filter (CUKF)}

3.1. State Model and Measurement Models of a Linear System. For linear systems, the state transfer model is [37]

$$
X(t)=F(t) X(t)+W(t)
$$

where $F$ can be established from system transfer models; $W$ is the system input noise, assumed to be white with zero mean. The state vector is defined as

$$
X=\left[\begin{array}{lllllllllll}
\delta L & \delta \lambda & \delta h & \delta V_{E} & \delta V_{N} & \delta V_{U} & \phi_{E} & \phi_{N} & \phi_{U} & \nabla_{b x} & \nabla_{b y}
\end{array}\right.
$$
$\left.\begin{array}{lllllll}\nabla_{b z} & \varepsilon_{b x} & \varepsilon_{b y} & \varepsilon_{b z} & M_{x} & M_{y} & M_{z}\end{array}\right]^{T}$ with $\delta L, \quad \delta \lambda$, and $\delta h$ denoting the latitude, longitude, and height position error in ENU axes, respectively; $\delta V_{E}, \delta V_{N}$, and $\delta V_{U}$ are the east, north, and upward velocity errors in ENU axes, respectively; $\phi_{E}, \phi_{N}$, and $\phi_{U}$ represent the heading, pitch, and roll errors in ENU axes, respectively; $\nabla_{b x}, \nabla_{b y}$, and $\nabla_{b z}$ set as the biases of the accelerometer projections onto the ENU axes; $\varepsilon_{b x}, \varepsilon_{b y}$, and $\varepsilon_{b z}$ for the gyroscope drift projections onto the ENU axes, respectively; $M_{x}, M_{y}$, and $M_{z}$ are the measured magnetic field vector in the ENU axes, respectively.

The linear measurement model is defined as

$$
Z(t)=\left[\begin{array}{c}
V_{E}^{\mathrm{INS}}-V_{E}^{\mathrm{DR}} \\
V_{N}^{\mathrm{INS}}-V_{N}^{\mathrm{DR}} \\
V_{U}^{\mathrm{INS}}-V_{U}^{\mathrm{DR}} \\
\varphi^{\mathrm{Gyro}}-\varphi^{\mathrm{Mag}} \\
\theta^{\mathrm{Gyro}}-\theta^{\mathrm{Acce}} \\
\gamma^{\text {Gyro }}-\gamma^{\text {Acce }}
\end{array}\right]=H(t) X(t)+V(t),
$$

where $V_{E}^{\mathrm{INS}}, V_{N}^{\mathrm{INS}}$, and $V_{U}^{\mathrm{INS}}$ are the estimated velocities by DR (Dead Reckoning) along the east, north, and upward direction, respectively; $V_{E}^{\mathrm{DR}}, V_{N}^{\mathrm{DR}}$, and $V_{U}^{\mathrm{DR}}$ represent the measurement velocity of INS (Inertial Navigation System) along the east, north, and upward direction, respectively. 
$\varphi^{\text {Gyro }}$ and $\varphi^{\text {Mag }}$ are the heading measured by gyroscopes and magnetometers, respectively; $\theta^{\text {Gyro }}$ and $\theta^{\text {Acce }}$ denote the pitch measured by gyroscopes and accelerometers, respectively; $\gamma^{\text {Gyro }}$ and $\gamma^{\text {Acce }}$ describe the roll measured by gyroscopes and accelerometers, respectively; $H$ is the observation matrix; $V$ is a white Gaussian measurement noise with zero mean value.

3.2. CUKF Models. Generally, for underwater navigation, the state and measurement models of multisensor fusion are nonlinear [38, 39]. How to derive a nonlinear sequential state estimation from equations (3) and (4) is the main task. One can draw the nonlinear state and measurement models as follows:

$$
\begin{gathered}
X_{k+1}=f\left(X_{k}\right)+W_{k}, \\
Z_{k}=h\left(X_{k}\right)+V_{k},
\end{gathered}
$$

where $X_{k}$ and $Z_{k}$ represent the state vector and measurement at time step $k$, respectively. $f(\cdot)$ is $n$-dimensional nonlinear function for state transition and $h(\cdot)$ is the $m$ dimensional nonlinear measurement function. $W_{k}$ and $V_{k}$ are zero mean process noise and measurement noise with covariance matrix $Q_{k}$ and $R_{k}$, respectively. Through discretization, it is straightforward to convert the process and measurement equations in (12) and (13) to the nonlinear process function $f(\cdot)$ and measurement function $h(\cdot)$ in (5) and (6).

The degree of nonlinearity in the practical underwater navigation is much bigger than the theoretical analysis, especially for MEMS-grade sensors [40, 41]. It may not achieve the desired effect for traditional UKF, and this paper proposes a new algorithm of cubature unscented Kalman filter (CUKF) considering the trade-off among different aspects to improve further accuracy, real-time, and stability.

The procedure for implementing the CUKF can be summarized as follows:

Step 1: Sample. The $n$-dimensional random variable $X_{k}$ with mean $\widehat{X}_{k}$ and covariance $\widehat{P}_{k}$ is approximated by sigma points selected using the following equations:

$$
\begin{aligned}
\chi_{i, k-1} & =\widehat{X}_{k-1}, \quad i=0, \\
\chi_{i, k-1} & =\widehat{X}_{k-1}+\left(a \sqrt{L \widehat{P}_{k-1}}\right)_{i}, \\
i & =1, \ldots, L, \\
\chi_{i, k-1} & =\widehat{X}_{k-1}-\left(a \sqrt{L \widehat{P}_{k-1}}\right)_{i-L}, \\
i & =L+1, \ldots, 2 L,
\end{aligned}
$$

where $a \in R$ is a turning parameter denoting the spread of the sigma points around $\widehat{X}_{k-1}$ and is often set to a small positive value. This parameter only affects errors caused by more than second-order matrices. The $\left(\sqrt{L \widehat{P}_{k-1}}\right)_{i}$ is the $i$ thcolumn of the matrix square root of $L \widehat{P}_{k-1}$ which is real symmetric positive definite matrix obtained through Cholesky resolution for $L \widehat{P}_{k-1}$. The sample points are composed of sigma points set $\left\{\chi_{i}\right\}, i=0, \ldots, 2 L$, and the corresponding weight with these sample points is described as $\omega_{0}=1-\left(1 / a^{2}\right), \omega_{i}=\left(1 / 2 L a^{2}\right),(i=1, \ldots, 2 L)$, $\omega_{i}$ is the weight of ithsigma point, and $\sum_{i=0}^{2 n} \omega_{i}=1$.

Step 2: The first prediction. Each point is instantiated through the process model to yield a set of transformed samples

$$
\chi_{i, k \mid k-1}=f_{k-1}\left(\chi_{i, k-1}\right)
$$

The predicted mean and covariance are computed as

$$
\begin{aligned}
\widehat{X}_{k \mid k-1} & =\sum_{i=0}^{2 L} \omega_{i} \chi_{i, k \mid k-1}, \\
\widehat{P}_{k \mid k-1} & =\sum_{i=0}^{2 L} \omega_{i}\left(\chi_{i, k \mid k-1}-\widehat{X}_{k \mid k-1}\right)\left(\chi_{i, k \mid k-1}-\widehat{X}_{k \mid k-1}\right)^{T}+Q_{k} .
\end{aligned}
$$

Step 3: The first update, with the process of measurement update, is as follows:

The measurement vector is

$$
Z_{k}=h_{k-1}\left(\chi_{i, k \mid k-1}\right)
$$

The first predicted measurement vector is

$$
\widehat{Z}_{k}=\sum_{i=0}^{2 n} \omega_{i} Z_{i, k \mid k-1} \text {. }
$$

The first covariance matrix of the innovations is

$\widehat{P}_{k}^{z z}=\sum_{i=0}^{2 n} \omega_{i}\left(Z_{i, k \mid k-1}-\widehat{Z}_{k}\right)\left(Z_{i, k k-1}-\widehat{Z}_{k}\right)^{T}+R_{k}$.

The first cross covariance matrix between the predicted state estimate errors and innovations is

$\widehat{P}_{k}^{x z}=\sum_{i=0}^{2 n} \omega_{i}\left(\chi_{i, k \mid k-1}-\widehat{X}_{k \mid k-1}\right)\left(Z_{i, k \mid k-1}-\widehat{Z}_{k}\right)^{T}$.

The gain matrix is

$$
K_{k}=\widehat{P}_{k}^{x z}\left(\widehat{P}_{k}^{z z}\right)^{-1}
$$

The updated state estimates are

$$
\widehat{X}_{k}=\widehat{X}_{k \mid k-1}+K_{k}\left(Z_{k}-\widehat{Z}_{k}\right)
$$

The covariance matrix of errors in the updated state estimates is

$$
\widehat{P}_{k}=\widehat{P}_{k \mid k-1}+K_{k} \widehat{P}_{z, k} K_{k}^{T} .
$$




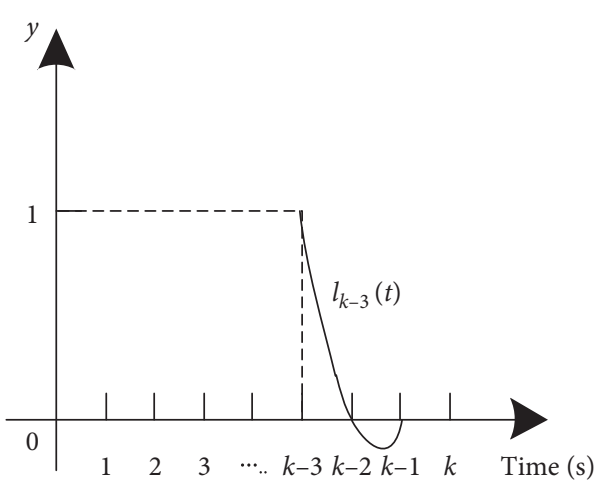

(a)

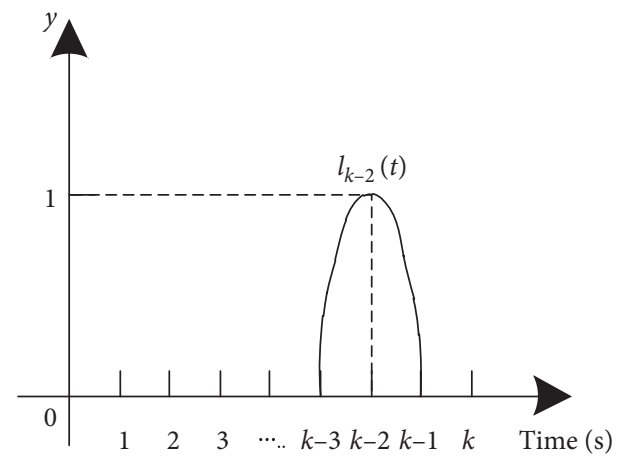

(b)

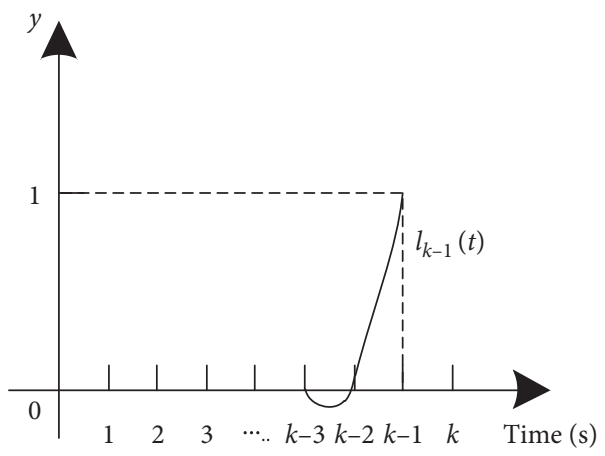

(c)

Figure 1: QI method from the time sequence 1 to $(k)-1$. (a) QI basis function at the time sequence $k-3$. (b) QI basis function at the time sequence $k-2$. (c) QI basis function at the time $k-1$.

Step 4: Cubature points generation

Update the covariance matrix of error

$$
\widehat{P}_{k}=S_{k} S_{k}^{T}
$$

Generate the cubature points

$$
\begin{aligned}
\xi_{j, k}=\left[\left(\xi_{j, k}^{1}\right)^{T}\left(\xi_{j, k}^{2}\right)^{T}\right]=A_{k} \xi_{j}+\widehat{X}_{k}, & \\
& j=1, \ldots, N .
\end{aligned}
$$

Calculate the propagated cubature points

$$
\begin{aligned}
& \mu_{j, k+1 \mid k}=f_{k}\left(\xi_{j, k}^{1}\right), \quad j=1, \ldots, N, \\
& \pi_{j, k+1 \mid k}=h_{k}\left(\xi_{j, k}^{1}\right), \quad j=1, \ldots, N,
\end{aligned}
$$

Step 5: The second prediction.

The predicted mean and covariance are calculated as

$$
\begin{aligned}
\widehat{X}_{k+1 \mid k}= & \frac{1}{2 N} \sum_{j=1}^{2 N} \mu_{j, k+1 \mid k}, \\
\widehat{P}_{k+1 \mid k}= & \frac{1}{2 N} \sum_{j=1}^{2 N} \mu_{j, k+1 \mid k}\left(\mu_{j, k+1 \mid k}\right)^{T} \\
& -\widehat{X}_{k+1 \mid k}\left(\widehat{X}_{k+1 \mid k}\right)^{T}+Q_{k} .
\end{aligned}
$$

Step 6: The second update.

Factorize

$$
\widehat{P}_{k+1 \mid k}=S_{k+1} S_{k+1}^{T} .
$$

Generate the cubature points

$$
\xi_{j, k+1}=A_{k+1} \xi_{j}+\widehat{X}_{k+1 \mid k}, \quad j=1, \ldots, 2 L .
$$

Calculate the propagated cubature points:

$$
\pi_{j, k+1 \mid k}^{*}=h_{k+1}\left(\xi_{j, k+1}\right), \quad j=1, \ldots, 2 L .
$$

The second predicted measurement

$$
\widehat{Z}_{k+1}=\frac{1}{2 L} \sum_{j=1}^{2 L} \pi_{j, k+1 \mid k}^{*} .
$$

The second covariance matrix of the innovations

$$
\begin{aligned}
& \widehat{P}_{k+1}^{z z}=\frac{1}{2 L} \sum_{j=1}^{2 L}\left(\pi_{j, k+1 \mid k}^{*}\right)\left(\pi_{j, k+1 \mid k}^{*}\right)^{T}-\widehat{Z}_{k+1} Z_{k+1}^{T}+R_{k+1}, \\
& \widehat{P}_{k+1}^{z z}=\frac{1}{2 L} \sum_{j=1}^{2 L}\left(\pi_{j, k+1 \mid k}^{*}\right)\left(\pi_{j, k+1 \mid k}^{*}\right)^{T}-\widehat{Z}_{k+1} Z_{k+1}^{T}+R_{k+1} .
\end{aligned}
$$


TABLE 1: The pseudocode of the proposed algorithm.

Pseudocode

Input: $\mathbf{X}_{0 \mid 0}, \mathbf{P}_{0 \mid 0}, \mathbf{Q}_{0}$

LIP method:

$$
\begin{aligned}
& \hat{Y}_{k}=\mathbf{Y}_{k-3} l_{k-3}\left(t_{k}\right)+\mathbf{Y}_{k-2} l_{k-2}\left(t_{k}\right)+\mathbf{Y}_{k-1} l_{k-1}\left(t_{k}\right) \\
& =\mathbf{Y}_{k-3}\left(\left(t_{k}-t_{k-2}\right)\left(t_{k}-t_{k-1}\right) /\left(t_{k-3}-t_{k-2}\right)\left(t_{k-3}-t_{k-1}\right)\right)+\mathbf{Y}_{k-2}\left(\left(t_{k}-t_{k-3}\right)\left(t_{k}-t_{k-1}\right) /\left(t_{k-2}-t_{k-3}\right)\left(t_{k-2}-t_{k-1}\right)\right) \\
& +\mathbf{Y}_{k-1}\left(\left(t_{k}-t_{k-3}\right)\left(t_{k}-t_{k-2}\right) /\left(t_{k-1}-t_{k-3}\right)\left(t_{k-1}-t_{k-2}\right)\right)
\end{aligned}
$$

Time update:

$\mathbf{X}_{k \mid k-1}=f_{k-1}\left(\mathbf{X}_{k-1 \mid k-1}\right)$

$\mathbf{P}_{k \mid k-1}=\left(\mathbf{X}_{k \mid k-1}-\mathbf{X}_{k-1 \mid k-1}\right)\left(\mathbf{X}_{k \mid k-1}-\mathbf{X}_{k-1 \mid k-1}\right)^{T}+\mathbf{Q}_{k-1}$

Variational Bayesian measurement update initialization:

$\mathbf{X}_{k \mid k}^{(0)}=\mathbf{X}_{k \mid k-1}, \mathbf{P}_{k \mid k-1}^{(0)}=\mathbf{P}_{k \mid k-1}, \mathbf{M}_{k \mid k-1}^{(0)}, m_{k \mid k-1}^{(0)}, \mathbf{U}_{k \mid k-1}^{(0)}, u_{k \mid k-1}^{(0)}$.

Variational Bayesian measurement estimation: for $l=0$ : NVB- 1

The parameters of $\mathbf{P}_{k \mid k-1}$ :

$\left\{\begin{array}{l}m_{k \mid k-1}^{(l+1)}=m_{k \mid k-1}^{(l)}+1 \\ \mathbf{M}_{k \mid k-1}^{(l+1)}=\mathbf{A}_{k}^{(l)}+\mathbf{M}_{k \mid k-1}^{(l)}\end{array}\right.$

The parameters of $\mathbf{R}_{k}$ :

$\left\{\begin{array}{l}u_{k \mid k-1}^{(l+1)}=u_{k \mid k-1}^{(l)}+1 \\ \mathbf{U}_{k \mid k-1}^{(l+1)}=\mathbf{B}_{k}^{(l)}+\mathbf{U}_{k \mid k-1}^{(l)}\end{array}\right.$

The expectations of $\mathbf{P}_{k \mid k-1}$ and $\mathbf{R}_{k}$ are calculated:

$E^{(l+1)}\left(\mathbf{P}_{k \mid k-1}\right)=\mathbf{M}_{k \mid k-1}^{(l+1)} /\left(m_{k \mid k-1}^{(l+1)}-n_{\mathbf{X}}-1\right)$

$E^{(l+1)}\left(\mathbf{R}_{k}\right)=\mathbf{U}_{k \mid k-1}^{(l+1)} /\left(u_{k \mid k-1}^{(l+1)}-n_{\widehat{Y}}-1\right)$

The state vector $\mathbf{X}_{k \mid k}^{(l+1)}$ and error covariance matrix $\mathbf{P}_{k \mid k}^{(l+1)}$ :

$\left\{\begin{array}{l}\mathbf{X}_{k \mid k}^{(l+1)}=\mathbf{X}_{k \mid k}^{(l)}+\mathbf{K}_{k}^{(l+1)}\left(\bar{Y}_{k}-\mathrm{H}_{k} \mathbf{X}_{k \mid k}^{(l)}\right) \\ \mathbf{P}_{k \mid k}^{(l+1)}=\mathbf{P}_{k \mid k-1}^{(l+1)}-\mathbf{K}_{k}^{(l+1)} \mathrm{H}_{k} \mathbf{P}_{k \mid k-1}^{(l+1)}\end{array}\right.$

end

Output: $\mathbf{X}_{k \mid k}, \mathbf{P}_{k \mid k}$.

TABLE 2: The simulation parameters.

\begin{tabular}{lc}
\hline Parameters & Values \\
\hline $\mathbf{P}_{0 \mid 0}$ & eye $(4)$ \\
$\mathbf{Q}_{k-1}$ & $10^{-3} \times \operatorname{diag}([1,0.5,1,0.5])$ \\
$\mathbf{R}_{k}$ & eye $(4)$ \\
\hline
\end{tabular}

Step 7: Compute gain matrix, estimate state, and its covariance matrix secondly:

$$
\begin{aligned}
& K_{k+1}=\widehat{P}_{k+1}^{x z}\left(\widehat{P}_{k+1}^{z z}\right)^{-1}, \\
& \widehat{X}_{k+1}=\widehat{X}_{k+1 \mid k}+K_{k+1}\left(Z_{k+1}-\widehat{Z}_{k+1}\right), \\
& \widehat{P}_{k+1}=\widehat{P}_{k+1 \mid k}+K_{k+1} \widehat{P}_{k+1}^{z z} K_{k+1}^{T} .
\end{aligned}
$$

Step 8: Repeat Steps 1 to 7 for the next sample.

\section{Quadratic Interpolation-Based Variational Bayesian Filter}

4.1. Lagrange Interpolation Polynomial. When the measurement information is lost, the Lagrange interpolation polynomial (LIP) can be used to calculate the predicted observed vector $Y_{k}$. The QI method is used because the Runge phenomenon needs to be avoided [42, 43].

Assumption 1. (a) The measurement information is lost at the time $k$. (b) The measurement information is observed from the time 1 to $\mathrm{k}-1$, as the method function in Figure 1 .

The QI polynomial is described as

$$
L_{2}(t)=\mathbf{Y}_{k-3} l_{k-3}(t)+\mathbf{Y}_{k-2} l_{k-2}(t)+\mathbf{Y}_{k-1} l_{k-1}(t),
$$

where $\quad l_{k-3}(t)=\left(\left(t-t_{k-2}\right)\left(t-t_{k-1}\right) /\left(t_{k-3}-t_{k-2}\right)\left(t_{k-3}\right.\right.$ $\left.\left.-t_{k-1}\right)\right), \quad l_{k-2}(t)=\left(\left(t-t_{k-3}\right)\left(t-t_{k-1}\right) /\left(t_{k-2}-t_{k-3}\right)\right.$ 


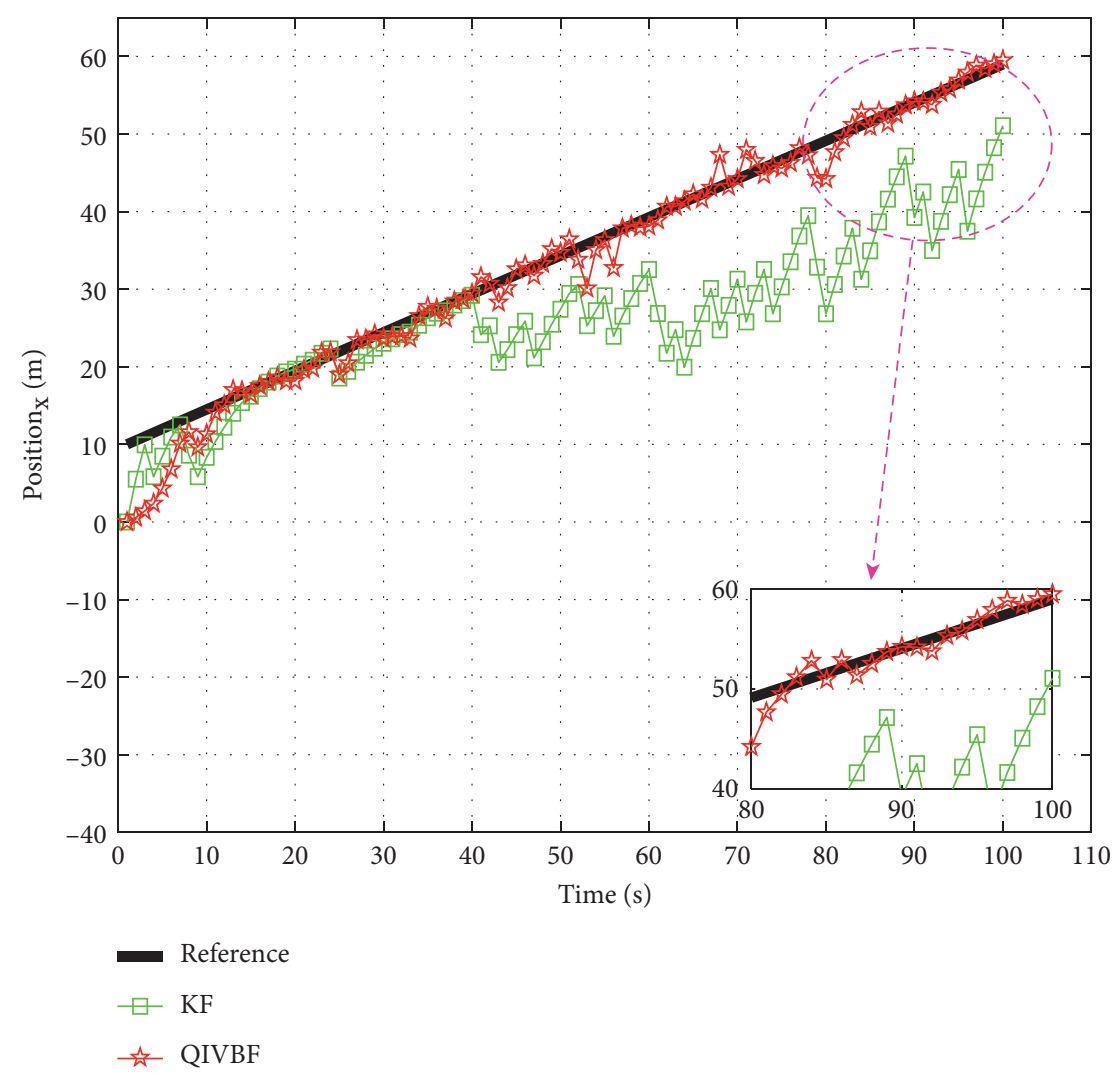

FIgure 2: The positions in $x$ direction of the different algorithms.

$\left.\left(t_{k-2}-t_{k-1}\right)\right)$, and $l_{k-1}(t)=\left(\left(t-t_{k-3}\right)\left(t-t_{k-2}\right) /\left(t_{k-1}-t_{k-3}\right)\right.$ $\left.\left(t_{k-1}-t_{k-2}\right)\right)$.

Hence, $Y_{k}$ can be factored as follows:

$$
\begin{aligned}
\widehat{Y}_{k}= & \mathbf{Y}_{k-3} l_{k-3}\left(t_{k}\right)+\mathbf{Y}_{k-2} l_{k-2}\left(t_{k}\right)+\mathbf{Y}_{k-1} l_{k-1}\left(t_{k}\right) \\
= & \mathbf{Y}_{k-3} \frac{\left(t_{k}-t_{k-2}\right)\left(t_{k}-t_{k-1}\right)}{\left(t_{k-3}-t_{k-2}\right)\left(t_{k-3}-t_{k-1}\right)} \\
& +\mathbf{Y}_{k-2} \frac{\left(t_{k}-t_{k-3}\right)\left(t_{k}-t_{k-1}\right)}{\left(t_{k-2}-t_{k-3}\right)\left(t_{k-2}-t_{k-1}\right)} \\
& +\mathbf{Y}_{k-1} \frac{\left(t_{k}-t_{k-3}\right)\left(t_{k}-t_{k-2}\right)}{\left(t_{k-1}-t_{k-3}\right)\left(t_{k-1}-t_{k-2}\right)}
\end{aligned}
$$

where $\widehat{Y}_{k}$ is the predicted observed vector at the time $k$ and $t_{k}$ means the time of $k$.

4.2. The Variational Bayesian Approach. In order to estimate the state vector $\mathbf{X}_{k \mid k}$ with the measurement information lost, the joint $\operatorname{PDF} \operatorname{PDF}\left(\mathbf{X}_{k \mid k}, \mathbf{P}_{k \mid k-1}, \mathbf{R}_{k}, \mathbf{Y}_{1: k-1}, \widehat{Y}_{k}\right)$ is considered to calculate the optimal solutions of $\mathbf{X}_{k \mid k}, \mathbf{P}_{k \mid k-1}$ and $\mathbf{R}_{k}$. However, this PDF cannot be obtained, so the VB method is used to find some independent PDFs $q(\cdot)$, which are used to approximate $\operatorname{PDF}\left(\mathbf{X}_{k \mid k}, \mathbf{P}_{k \mid k-1}, \mathbf{R}_{k}, \mathbf{Y}_{1: k-1}, \widehat{Y}_{k}\right)$.

Set $\Theta=\left\{\mathbf{X}_{k|| k}, \mathbf{P}_{k \mid k-1}, \mathbf{R}_{k}\right\}$. Then, the optimal solutions of $\Theta$, which is satisfied, are as follows:

$$
\log q(\kappa)=E_{\Theta^{-\kappa}}\left\{\log \left[\operatorname{PDF}\left(\Theta, \mathbf{Y}_{1: k-1}, \hat{Y}_{k}\right)\right]\right\}+C_{\kappa}
$$

where $E_{\Theta^{-\kappa}}\left\{\log \left[\operatorname{PDF}\left(\Theta, \mathbf{Y}_{1: k-1}, \widehat{Y}_{k}\right)\right]\right\}$ is the expectation $\log \left[\operatorname{PDF}\left(\Theta, \mathrm{Y}_{1: k-1}, \widehat{Y}_{k}\right)\right]$ of the $\Theta$ except for $\kappa . C_{\kappa}$ is a constant about $\kappa$. In Bayesian statistics theory, the covariance matrix is defined to follow the inverse Wishart (IW) distribution.

The distributions of $\mathbf{P}_{k \mid k-1}$ and $\mathbf{R}_{k}$ are set in

$$
\left\{\begin{array}{l}
q\left(\mathbf{P}_{k \mid k-1}\right)=\operatorname{IW}\left(\mathbf{P}_{k \mid k-1} ; m_{k \mid k-1}, \mathbf{M}_{k \mid k-1}\right), \\
q\left(\mathbf{R}_{k}\right)=\operatorname{IW}\left(\mathbf{R}_{k} ; u_{k \mid k-1}, \mathbf{U}_{k \mid k-1}\right),
\end{array}\right.
$$

where IW $\left(\mathbf{P}_{k \mid k-1} ; m_{k \mid k-1}, \mathbf{M}_{k \mid k-1}\right)$ is the variable $\mathbf{P}_{k \mid k-1}$ that follows IW distribution with $m_{k \mid k-1}$ degree of freedom (DOF) and $\mathbf{M}_{k \mid k-1}$ inverse scale matrix. IW $\left(\mathbf{R}_{k} ; u_{k \mid k-1}, \mathbf{U}_{k \mid k-1}\right)$ is the variable $\mathbf{R}_{k}$ that follows IW distribution with $u_{k \mid k-1}$ DOF and $\mathbf{U}_{k \mid k-1}$ inverse scale matrix; $\log \left[\operatorname{PDF}\left(\Theta, \mathbf{Y}_{1: k-1}, \bar{Y}_{k}\right)\right]$ can be rewritten as follows: 


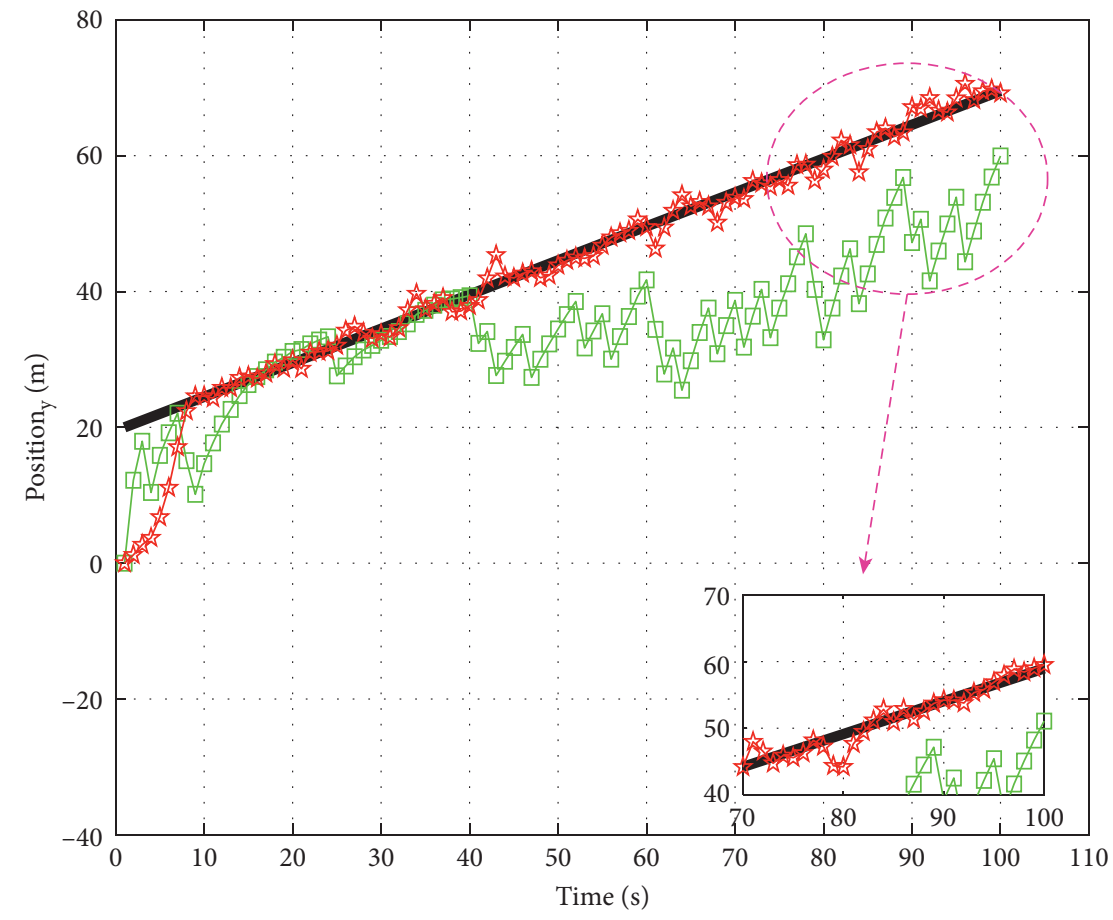

- Reference

$\square \mathrm{KF}$

年 $\mathrm{QIVBF}$

FIGURE 3: The positions in $y$ direction of the different algorithms.

$$
\begin{aligned}
\operatorname{PDF}\left(\Theta, \mathbf{Y}_{1: k-1}, \widehat{Y}_{k}\right)= & \operatorname{PDF}\left(\widehat{Y}_{k} \mid \mathbf{X}_{k \mid k}, \mathbf{R}_{k}\right) \operatorname{PDF}\left(\mathbf{X}_{k \mid k} \mid \mathbf{Y}_{1: k-1}, \mathbf{P}_{k \mid k-1}\right) \\
& \times \operatorname{IW}\left(\mathbf{P}_{k \mid k-1} ; m_{k \mid k-1}, \mathbf{M}_{k \mid k-1}\right) \operatorname{IW}\left(\mathbf{R}_{k} ; u_{k \mid k-1}, \mathbf{U}_{k \mid k-1}\right) \operatorname{PDF}\left(\mathbf{Y}_{1: k-1}\right) .
\end{aligned}
$$

Hence, $\operatorname{PDF}\left(\Theta, \mathbf{Y}_{1: k-1}, \hat{Y}_{k}\right)$ is expended in

$$
\begin{aligned}
\operatorname{PDF}\left(\Theta, \mathbf{Y}_{1: k-1}, \widehat{Y}_{k}\right)= & N\left(\hat{Y}_{k} ; h_{k}\left(\mathbf{X}_{k \mid k}\right), \mathbf{R}_{k}\right) N\left(\mathbf{X}_{k \mid k} ; \mathbf{X}_{k \mid k-1}, \mathbf{P}_{k \mid k-1}\right) \\
& \times \operatorname{IW}\left(\mathbf{P}_{k \mid k-1} ; m_{k \mid k-1}, \mathbf{M}_{k \mid k-1}\right) \operatorname{IW}\left(\mathbf{R}_{k} ; u_{k \mid k-1}, \mathbf{U}_{k \mid k-1}\right) \operatorname{Pro}\left(\mathbf{Y}_{1: k-1}\right) .
\end{aligned}
$$

The optimal solution is defined as follows:

$$
\begin{aligned}
\log \left[\operatorname{PDF}\left(\Theta, \mathbf{Y}_{1: k-1}, \widehat{Y}_{k}\right)\right]= & -\frac{1}{2}\left(\widehat{Y}_{k}-\mathrm{H}_{k} \mathbf{X}_{k \mid k}^{(l)}\right)^{T} \mathbf{R}_{k}^{-1}\left(\hat{Y}_{k}-\mathrm{H}_{k} \mathbf{X}_{k \mid k}^{(l)}\right) \\
& -\frac{1}{2}\left(\mathbf{X}_{k \mid k}-\mathbf{X}_{k \mid k-1}\right)^{T} \mathbf{P}_{k \mid k-1}^{-1}\left(\mathbf{X}_{k \mid k}-\mathbf{X}_{k \mid k-1}\right)-\frac{1}{2}\left(n_{\widehat{Y}}+u_{k \mid k-1}+2\right) \log \mid \mathbf{R}_{k} \\
& -\frac{1}{2} \operatorname{tr}\left(\mathbf{U}_{k \mid k-1} \mathbf{R}_{k}^{-1}\right)-\frac{1}{2}\left(n_{\mathbf{X}}+m_{k \mid k-1}+2\right) \log \left|\mathbf{P}_{k \mid k-1}\right|-\frac{1}{2} \operatorname{tr}\left(\mathbf{M}_{k \mid k-1} \mathbf{P}_{k \mid k-1}^{-1}\right)+C_{\kappa} .
\end{aligned}
$$

When choosing $\kappa=\mathbf{P}_{k \mid k-1}$, the parameters of $\mathbf{P}_{k \mid k-1}$ are given by using

$$
\left\{\begin{array}{l}
m_{k \mid k-1}^{(l+1)}=m_{k \mid k-1}^{(l)}+1, \\
\mathbf{M}_{k \mid k-1}^{(l+1)}=\mathbf{A}_{k}^{(l)}+\mathbf{M}_{k \mid k-1}^{(l)},
\end{array}\right.
$$




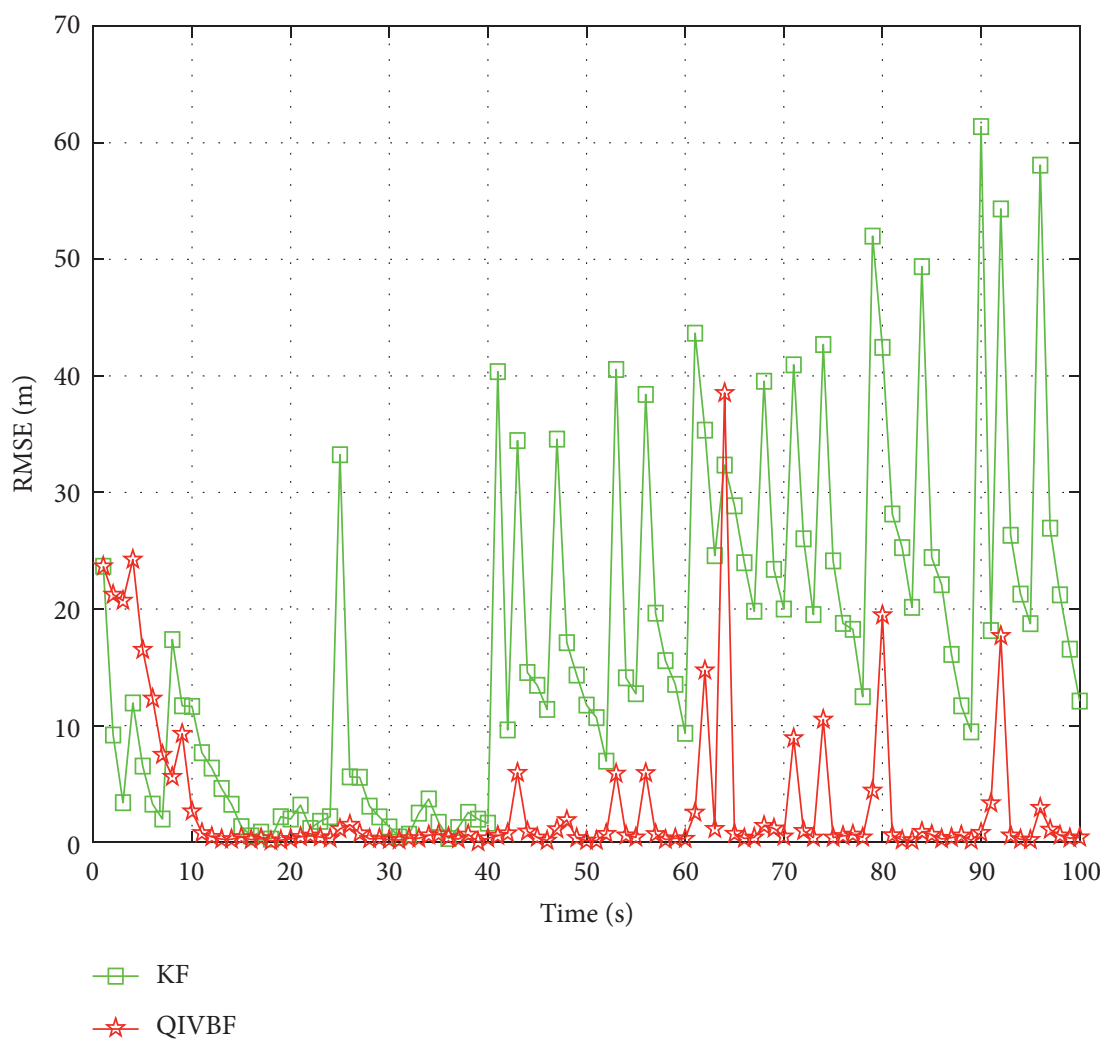

FIgURE 4: RMSE of the KF and QIVBF algorithms.

where $\mathbf{A}_{k}^{(l)}=\mathbf{P}_{k \mid k-1}+\left(\mathbf{X}_{k \mid k}^{(l)}-\mathbf{X}_{k \mid k-1}\right)\left(\mathbf{X}_{k \mid k}^{(l)}-\mathbf{X}_{k \mid k-1}\right)^{T}$.

When choosing $\kappa=\mathbf{R}_{k}$, the parameters of $\mathbf{R}_{k}$ are given by using

$$
\left\{\begin{array}{l}
u_{k \mid k-1}^{(l+1)}=u_{k \mid k-1}^{(l)}+1, \\
\mathbf{U}_{k \mid k-1}^{(l+1)}=\mathbf{B}_{k}^{(l)}+\mathbf{U}_{k \mid k-1}^{(l)},
\end{array}\right.
$$

where $\mathbf{B}_{k}^{(l)}$ is calculated as follows:

$$
\mathbf{B}_{k}^{(l)}=E^{(l)}\left[\left(\widehat{Y}_{k}-\mathrm{H}_{k} \mathbf{X}_{k \mid k}^{(l)}\right)\left(\widehat{Y}_{k}-\mathrm{H}_{k} \mathbf{X}_{k \mid k}^{(l)}\right)^{T}\right]
$$

Then, $\mathbf{P}_{k \mid k-1}$ and $\mathbf{R}_{k}$ at $l+1$ th step are calculated as follows, respectively:

$$
\begin{aligned}
E^{(l+1)}\left(\mathbf{P}_{k \mid k-1}\right) & =\frac{\mathbf{M}_{k \mid k-1}^{(l+1)}}{\left(m_{k \mid k-1}^{(l+1)}-n_{\mathbf{X}}-1\right)}, \\
E^{(l+1)}\left(\mathbf{R}_{k}\right) & =\frac{\mathbf{U}_{k \mid k-1}^{(l+1)}}{\left(u_{k \mid k-1}^{(l+1)}-n_{\widehat{Y}}-1\right)} .
\end{aligned}
$$

When choosing $\kappa=\mathbf{X}_{k \mid k}, q\left(\mathbf{X}_{k \mid k}\right)$ is modelled as follows:

$$
q\left(\mathbf{X}_{k \mid k}\right)=N\left(\mathbf{X}_{k \mid k} ; \mathbf{X}_{k \mid k}^{(l+1)}, \mathbf{P}_{k \mid k}^{(l+1)}\right),
$$

where $N\left(\mathbf{X}_{k \mid k} ; \mathbf{X}_{k \mid k}^{(l+1)}, \mathbf{P}_{k \mid k}^{(l+1)}\right)$ denotes the variable $\mathbf{X}_{k \mid k}$ that follows Gaussian distribution with $\mathbf{X}_{k \mid k}^{(l+1)}$ mean vector and $\mathbf{P}_{k \mid k}^{(l+1)}$ error covariance matrix, and the measurement update is written as follows:

$$
\left\{\begin{array}{l}
\mathbf{X}_{k \mid k}^{(l+1)}=\mathbf{X}_{k \mid k}^{(l)}+\mathbf{K}_{k}^{(l+1)}\left(\widehat{Y}_{k}-\mathrm{H}_{k} \mathbf{X}_{k \mid k}^{(l)}\right), \\
\mathbf{P}_{k \mid k}^{(l+1)}=\mathbf{P}_{k \mid k-1}^{(l+1)}-\mathbf{K}_{k}^{(l+1)} \mathrm{H}_{k} \mathbf{P}_{k \mid k-1}^{(l+1)},
\end{array}\right.
$$

where the Kalman gain $\mathbf{K}_{k}^{(l+1)}$ at the $l+1$ th step is calculated by using (41) at time $k$.

$$
\mathbf{K}_{k}^{(l+1)}=\mathbf{P}_{k \mid k-1}^{(l+1)} \mathbf{H}_{k}^{T}\left[\mathbf{H}_{k} \mathbf{P}_{k \mid k-1}^{(l+1)} \mathbf{H}_{k}^{T}+\mathbf{R}_{k}^{(l+1)}\right]^{-1} .
$$

After fixed-point iteration $N_{\mathrm{VB}}$, the $\mathrm{VB}$ approximate of $\mathrm{PDF}$ is derived by

$$
\begin{gathered}
q\left(\mathbf{P}_{k \mid k-1}\right) \approx q^{\left(N_{\mathrm{VB}}\right)}\left(\mathbf{P}_{k \mid k-1}\right)=\operatorname{IW}\left(\mathbf{P}_{k \mid k-1} ; m_{k \mid k-1}^{\left(N_{\mathrm{VB}}\right)}, \mathbf{M}_{k \mid k-1}^{\left(N_{\mathrm{VB}}\right)}\right), \\
q\left(\mathbf{R}_{k}\right) \approx q^{\left(N_{\mathrm{VB}}\right)}\left(\mathbf{R}_{k}\right)=\operatorname{IW}\left(\mathbf{R}_{k} ; u_{k \mid k-1}^{\left(N_{\mathrm{VB}}\right)}, \mathbf{U}_{k \mid k-1}^{\left(N_{\mathrm{VB}}\right)}\right), \\
q\left(\mathbf{X}_{k \mid k}\right) \approx q^{\left(N_{\mathrm{VB}}\right)}\left(\mathbf{X}_{k \mid k}\right)=N\left(\mathbf{X}_{k \mid k} ; \mathbf{X}_{k \mid k}^{\left(N_{\mathrm{VB}}\right)}, \mathbf{P}_{k \mid k}^{\left(N_{\mathrm{VB}}\right)}\right) .
\end{gathered}
$$

The pseudocode is presented in Table 1. If the measurement information vector is lost, the LIP method is used to calculate the predicted observed vector $\widehat{Y}_{k}$. Then, the predicted state vector $\mathbf{X}_{k \mid k-1}$ and predicted error covariance matrix $\mathbf{P}_{k \mid k-1}$ are calculated by using the state vector $\mathbf{X}_{k \mid k}$, error covariance matrix $\mathbf{P}_{k \mid k}$ and process noise matrix $\mathbf{Q}_{k}$. Then, $\mathbf{X}_{k \mid k}^{(0)}, \mathbf{P}_{k \mid k-1}^{(0)}, \mathbf{M}_{k \mid k-1}^{(0)}, m_{k \mid k-1}^{(0)}, \mathbf{U}_{k \mid k-1}^{(0)}$ and $u_{k \mid k-1}^{(0)}$ are initialized for the subsequent variational Bayesian measurement estimation. The predicted error covariance matrix 
$\mathbf{P}_{k \mid k-1}^{(l+1)}$ and the measurement noise matrix $\mathbf{R}_{k}^{(l+1)}$ at the $l+1$ th step are calculated in order to estimate the state vector $\mathbf{X}_{k \mid k}^{(l+1)}$ and error covariance matrix $\mathbf{P}_{k \mid k}^{(l+1)}$ at the $l+1$ th step, respectively. Finally, after $N_{\mathrm{VB}}$ th iteration, the accurate state vector $\mathbf{X}_{k \mid k}$ and error covariance matrix $\mathbf{P}_{k \mid k}$ are determined at the time $k$.

\section{Simulations and Results}

The proposed QIVBF is compared with the traditional KF in a simulation with measurement missing scenarios, and the root mean squared error (RMSE) is defined to compare the performances of these algorithms.

$$
\operatorname{RMSE}(k)=\sqrt{\left(P_{x, k}-X_{k}^{t}\right)^{2}+\left(P_{y, k}-Y_{k}^{t}\right)^{2}}
$$

where $\left(P_{x, k}, P_{y, k}\right)$ and $\left(\mathrm{X}_{k}^{t}, \mathrm{Y}_{k}^{t}\right)$ are the predicted position and reference position, respectively. The parameters are set in Table 2.

According to the navigation system, the accuracy of the methods is compared, and the above position estimation errors are analyzed. From Figures 2 and 3, the proposed QIVBF performs closer to the reference than the traditional KF. The proposed QIVBF results in a much better estimation accuracy than the existing filter on handling the problems of underwater navigation in the scenarios of measurement information missing. In particular, KF can easily lose tracking of the reference values by reason that the accumulated errors of iteration. On the other hand, the quadratic interpolation improves the observation vector for the precision and stability of estimation iterations when the environments change, or the measurement information is lost. Therefore, the results demonstrate that the proposed QIVBF outperforms the traditional KF in the condition of measurement information missing.

The navigation accuracy and anti-interference ability of the KF and QIVBF are compared by calculating the rootmean-square error (RMSE) of the position estimation. The RMSE has a good reflection of the measurement precision. It can be seen from Figure 4 that the QIVBF performs better than the traditional KF. When the measurement information is missing, the traditional KF cannot estimate the accurate Kalman gain, which leads to the filter divergence. However, the proposed QIVBF uses the QI method to estimate the lost observed vector, which can help estimate the accurate information about cross error covariance matrix. Then, the VB method can help calculate the accurate state vector and error covariance matrix. The RMSE of QIVBF is lower than KF. Hence, the performance of QIVBF is better than KF.

\section{Conclusions}

The paper proposes a quadratic interpolation-based variational Bayesian filter for underwater navigation when measurement information is lost, where the quadratic interpolation method is used to calculate the observed vector when the measurement information is lost. Then, the state vector together with the predicted error covariance matrix and the measurement noise matrix is estimated based on the VB method. Simulation results demonstrated the effectiveness of the proposed QIVBF algorithm as compared with the traditional algorithm in the case of measurement information missing. The idea of the proposed QIVBF can be extended to design a nonlinear filter for the non-Gaussian noises with measurement information lost. In future work, we plan to apply multiple datasets of underwater navigation measurements, so that the position and attitude can be estimated based on the fusion of various data sources for better accuracy.

\section{Data Availability}

The data used to support the findings of this study are available from the corresponding author upon request.

\section{Conflicts of Interest}

The authors declare that they have no conflicts of interest.

\section{Acknowledgments}

This work was funded by the projects of the National Natural Science Foundation of China (Grant nos. 62073078 and 61802428).

\section{References}

[1] C. C. Eriksen, T. J. Osse, R. D. Light et al., "Seaglider: a longrange autonomous underwater vehicle for oceanographic research," IEEE Journal of Oceanic Engineering, vol. 26, no. 4, pp. 424-436, 2001.

[2] C. R. Walker, J. Q. Stringfield, E. T. Wolbrecht et al., "Measurement of the magnetic signature of a moving surface vessel with multiple magnetometer-equipped AUV's," Ocean Engineering, vol. 64, no. 64, pp. 80-87, 2013.

[3] Y. Qing, S. Hao, T. Gong-You et al., "Optimal disturbances rejection control for autonomous underwater vehicles in shallow water environment," Mathematical Problems in Engineering, vol. 2017, Article ID 8506381, 9 pages, 2017.

[4] S. P. Hsu and T. S. Liu, "Modifications of control loop to improve the depth response of autonomous underwater vehicles," Mathematical Problems in Engineering, vol. 2014, Article ID 324813, 12 pages, 2014.

[5] D. Wang, L. X. Xu, and L. Hou, "An improved adaptive kalman filter for underwater SINS/DVL system," Mathematical Problems in Engineering, vol. 2020, Article ID 5324349, 14 pages, 2020.

[6] H. Huang, X. Chen, and J. Zhang, "Weight self-adjustment Adams implicit filtering algorithm for attitude estimation applied to underwater gliders," IEEE Access, vol. 4, no. 1, pp. 5695-5709, 2016.

[7] H. Q. Huang, X. Y. Chen, Z. K. Zhou, H. Liu, and C. P. Lv, "Study on INS/DR integration navigation system using EKF/ RK4 algorithm for underwater gliders," Journal of Marine Science and Technology, vol. 25, no. 1, pp. 84-95, 2017.

[8] T. Jinwu, X. Xu, L. Hou et al., "An ultra-short baseline positioning model based on rotating array \& reusing elements and its error analysis," Sensors, vol. 19, no. 20, p. 4373, 2019.

[9] Study on installation error analysis and calibration of acoustic transceiver array based on SINS/USBL integrated system. 
[10] S. A. Jenkins and G. D'Spain, Autonomous Underwater Glider, Springer Handbook of Ocean Engineering Springer, Dordrecht, Netherlands, 2016.

[11] X. Yu and J. Jiang, "Hybrid fault-tolerant flight control system design against partial actuator failures," IEEE Transactions on Control Systems Technology, vol. 20, no. 4, pp. 871-886, 2012.

[12] M. Joerger and B. Pervan, "Kalman filter-based integrity monitoring against sensor faults," Journal of Guidance, Control, and Dynamics, vol. 36, no. 2, pp. 349-361, 2013.

[13] H. Huang, J. Tang, B. Zhang, J. Chen, J. Zhang, and X. Song, "A novel nonlinear algorithm for non-Gaussian noises and measurement information loss in underwater navigation," IEEE Access, vol. 8, no. 8, pp. 118472-118484, 2020.

[14] H. Cao, R. Xue, Q. Cai et al., "Design and experiment for dualmass MEMS gyroscope sensing closed-loop system," IEEE Access, vol. 8, no. 8, pp. 48074-48087, 2020.

[15] H. Huang, J. Zhou, J. Zhang et al., "Attitude estimation fusing quasi-Newton and cubature Kalman filtering for inertial navigation system aided with magnetic sensors," IEEE Access, vol. 6, no. 6, pp. 28755-28767, 2018.

[16] H. Huang, X. Chen, B. Zhang, and J. Wang, "High accuracy navigation information estimation for inertial system using the multi-model EKF fusing adams explicit formula applied to underwater gliders," ISA Transactions, vol. 66, no. 66, pp. 414-424, 2017.

[17] Y. Yao, X. Xu, Y. Li, and T. Zhang, "A hybrid IMM based INS/ DVL integration solution for underwater vehicles," IEEE Transactions on Vehicular Technology, vol. 68, no. 6, pp. 5459-5470, 2019.

[18] N. Sahu, P. Babu, A. Kumar, and R. Bahl, "A novel algorithm for optimal placement of multiple inertial sensors to improve the sensing accuracy," IEEE Transactions on Signal Processing, vol. 68 , no. 68 , pp. $142-154,2020$.

[19] L. Yang, Z. Fubin, X. Demin et al., "Liveness-based RRT algorithm for autonomous underwater vehicles motion planning," Journal of Advanced Transportation, vol. 2017, Article ID 7816263, 10 pages, 2017.

[20] H. Q. Huang, R. D. Shi, J. Zhou et al., “Attitude determination method integrating square-root cubature Kalman filter with expectation-maximization for inertial navigation system applied to underwater glider," Review of Scientific Instruments, vol. 90, Article ID 095001, 2019.

[21] Y. Yao, X. Xu, D. Yang, and X. Xu, "An IMM-UKF aided SINS/USBL calibration solution for underwater vehicles," IEEE Transactions on Vehicular Technology, vol. 69, no. 4, pp. 3740-3747, 2020.

[22] X. Xu, Y. Sun, J. Gui, Y. Yao, and T. Zhang, "A fast robust inmotion alignment method for SINS with DVL aided," IEEE Transactions on Vehicular Technology, vol. 69, no. 4, pp. 3816-3827, 2020.

[23] Y. Huang, Y. Zhang, B. Xu, Z. Wu, and J. Chambers, "A new outlier-robust student's t based Gaussian approximate filter for cooperative localization," IEEE/ASME Transactions on Mechatronics, vol. 22, no. 5, pp. 2380-2386, 2017.

[24] H. Cao, Y. Zhang, Z. Han et al., "Pole-zero temperature compensation circuit design and experiment for dual-mass MEMS gyroscope bandwidth expansion," IEEE/ASME Transactions on Mechatronics, vol. 24, no. 2, pp. 677-688, 2019.

[25] Z. Zuo and B. Yang, "Study of simulation platform for BDS/ INS deep integration navigation," Mathematical Problems in Engineering, vol. 18, 2019.

[26] H. L. Cao, Y. J. Zhang, C. Shen, Y. Liu, and X. W. Wang, "Temperature energy influence compensation for MEMS vibration gyroscope based on RBF-NN-GA-KF method'," Shock and Vibration, vol. 2018, Article ID 2830686, 10 pages, 2018.

[27] G. Hu, B. Gao, Y. Zhong et al., "Unscented kalman filter with process noise covariance estimation for vehicular ins/gps integration system," Information Fusion, vol. 37, 2020.

[28] Y. Li, L. Hou, Y. Yang, and J. Tong, "Huber's M-estimationbased cubature kalman filter for an INS/DVL integrated system," Mathematical Problems in Engineering, vol. 202012 pages, Article ID 1060672, 2020.

[29] M. Wang and J.-C. Liu, "Bayesian semiparametric double autoregressive modeling," Mathematical Problems in Engineering, vol. 2019, no. 1, 9 pages, 2019.

[30] C. W. Fox and S. J. Roberts, "A tutorial on Variational Bayesian inference," Artificial Intelligence Review, vol. 38, no. 2, pp. 1-11, 2012.

[31] X. R. Li and Y. B. Shalom, "Multiple-model estimation with variable structure," IEEE Transactions on Automatic Control, vol. 41, no. 4, pp. 478-493, 1996.

[32] S. Sarkka and J. Hartikainen, "Non-linear noise adaptive kalman filtering via variational bayes," in Proceedings of the IEEE International Workshop on Machine Learning for Signal Processing, Southampto, UK, September 2013.

[33] X. Gao, J. Chen, D. Tao, and X. Li, "Multi-sensor centralized fusion without measurement noise covariance by variational bayesian approximation," IEEE Transactions on Aerospace and Electronic Systems, vol. 47, no. 1, pp. 718-272, 2011.

[34] P. Dong, H. Z. Jing, and K. Shen, "Variational bayesian adaptive cubature information filter based on Wishart distribution," IEEE Transactions on Automatic Control, vol. 62, no. 11, pp. 6051-6057, 2017.

[35] G. Hu, Y. W. Wang, and C. B. GuGao, "A new direct filtering approach to INS/GNSS integration," Aerospace Science and Technology, vol. 77, pp. 755-764, 2018.

[36] X. L. Gan, "State-space measurement update for GNSS/INS integrated navigation," Mathematical Problems in Engineering, vol. 2020, Article ID 3675824, 17 pages, 2020.

[37] G. Sun, R. Longman, and Z. Chen, "Observer kalman filter identification of suspen-dome," Mathematical Problems in Engineering, vol. 2017, Article ID 1601534, 9 pages, 2017.

[38] G. Xue, Y. S. Gao, and Y. Zhong, "A derivative UKF for tightly coupled INS/GPS integrated navigation," ISA Transactions, vol. 56, pp. 135-144, 2015.

[39] G. Hu, L. Ni, B. Gao et al., "Model predictive based unscented kalman filter for hypersonic vehicle navigation with INS/ GNSS integration,” IEEE Access, vol. 99, 2019.

[40] H. M. Ouakad, A. M. Alofi, and A. H. Nayfeh, "Dynamic analysis of multilayers based MEMS resonators," Mathematical Problems in Engineering, vol. 2017, Article ID 1262650, 14 pages, 2017.

[41] S. Łuczak, R. Grepl, and M. Bodnicki, "Selection of MEMS accelerometers for tilt measurements," Journal of Sensors, vol. 201718 pages, Article ID 9796146, 2017.

[42] Le Zou, L. Song, X. Wang et al., "A new approach to Newtontype polynomial interpolation with parameters," Mathematical Problems in Engineering, vol. 17, pp. 1024-123X, 2020.

[43] R. He and L. Zhang, "Interval recognition algorithm of the pavement surface condition based on Lagrange interpolation method," Mathematical Problems in Engineering, vol. 2020, no. 1, 8 pages, Article ID 4251027, 2020. 